\title{
A DECAM SEARCH FOR AN OPTICAL COUNTERPART TO THE LIGO GRAVITATIONAL-WAVE EVENT GW151226
}

P. S. Cowperthwaite ${ }^{1,57}$, E. Berger ${ }^{1}$, M. Soares-Santos ${ }^{2}$, J. Annis ${ }^{2}$, D. Brout ${ }^{3}$, D. A. Brown ${ }^{4}$, E. Buckley-Geer ${ }^{2}$, S. B. Cenko ${ }^{5,6}$, H. Y. Chen $^{7}$, R. Chornock ${ }^{8}$, H. T. Diehl ${ }^{2}$, Z. Doctor ${ }^{7}$, A. Drlica-Wagner ${ }^{2}$, M. R. Drout ${ }^{1}$, B. Farr $^{9}$, D. A. Finley ${ }^{2}$, R. J. Foley ${ }^{10,11,12}$, W. Fong ${ }^{13}$, D. B. Fox ${ }^{14}$, J. Frieman ${ }^{2,7}$, J. Garcia-Bellido ${ }^{15}$, M. S. S. Gill ${ }^{16,17}$, R. A. Gruendil ${ }^{11,18}$, K. Herner ${ }^{2}$, D. E. Holz ${ }^{9}$, D. Kasen ${ }^{19,20}$, R. Kessler ${ }^{7}$, H. Lin $^{2}$, R. Margutti ${ }^{21}$, J. Marriner ${ }^{2}$, T. Matheson ${ }^{22}$, B. D. MetzGer ${ }^{23}$, E. H. Neilsen JR. ${ }^{2}$, E. QuataerT ${ }^{24}$, A. Rest ${ }^{25}$, M. Sako ${ }^{3}$, D. Scolnic ${ }^{7}$, N. Smith ${ }^{13}$, F. Sobreira ${ }^{26,27}$, G. M. Strampelli ${ }^{25}$, V. A. Villar ${ }^{1}$, A. R. Walker ${ }^{28}$, W. Wester ${ }^{2}$, P. K. G. Williams ${ }^{1}$, B. Yanny ${ }^{2}$, T. M. C. Abbott $^{28}$, F. B. Abdalla ${ }^{29,30}$, S. Allam $^{2}$, R. Armstrong ${ }^{31}$, K. Bechtol ${ }^{32}$, A. Benoit-Lévy 29,33,34, E. Bertin ${ }^{33,34}$, D. Brooks ${ }^{29}$, D. L. Burke ${ }^{16,17}$, A. Carnero Rosell ${ }^{27,35}$, M. Carrasco Kind ${ }^{11,18}$, J. Carretero ${ }^{36,37}$, F. J. Castander ${ }^{36}$, C. E. Cunha ${ }^{16}$, C. B. D’Andrea ${ }^{38,39}$, L. N. Da Costa ${ }^{27,35}$, S. Desai ${ }^{40,41}$, J. P. Dietrich ${ }^{40,41}$, A. E. Evrard ${ }^{42,43}$, A. Fausti Neto $^{27}$, P. Fosalba ${ }^{36}$, D. W. Gerdes ${ }^{42,43}$, T. Giannantonio ${ }^{44,45}$, D. A. Goldstein ${ }^{20,46}$, D. GRuen $^{16,17}$, G. Gutierrez ${ }^{2}$, K. Honscheid ${ }^{47,48}$, D. J. James ${ }^{28}$, M. W. G. Johnson ${ }^{18}$, M. D. Johnson ${ }^{18}$, E. Krause ${ }^{16}$, K. Kuehn ${ }^{49}$, N. Kuropatkin ${ }^{2}$, M. Lima ${ }^{27,50}$, M. A. G. Maia ${ }^{27,35}$, J. L. Marshall ${ }^{51}$, F. Menanteau ${ }^{11,18}$, R. Miquel ${ }^{37,52}$, J. J. Mohr ${ }^{40,41,53}$,

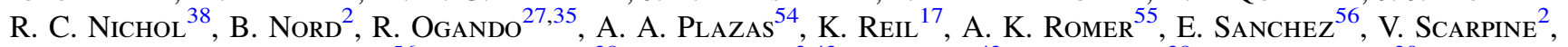
I. Sevilla-Noarbe ${ }^{56}$, R. C. Smith ${ }^{28}$, E. Suchyta ${ }^{3,43}$, G. Tarle ${ }^{43}$, D. Thomas ${ }^{38}$, R. C. Thomas ${ }^{20}$, D. L. TUCKER ${ }^{2}$, AND J. WELLER ${ }^{41,53,57}$

(The DES Collaboration)

${ }^{1}$ Harvard-Smithsonian Center for Astrophysics, 60 Garden Street, Cambridge, MA 02138, USA; pcowpert @cfa.harvard.edu

${ }^{2}$ Fermi National Accelerator Laboratory, P.O. Box 500, Batavia, IL 60510, USA

${ }^{3}$ Department of Physics and Astronomy, University of Pennsylvania, Philadelphia, PA 19104, USA

${ }^{5}$ Astrophysics Science Division, NASA Goddard Space Flight Center, 8800 Greenbelt Road, Greenbelt, MD 20771, USA
${ }_{6}$ Physics Department, Syrace Univeris, Syracuse, NY 1324, USA

6 Joint Space-Science Institute, University of Maryland, College Park, MD 20742, USA

${ }^{7}$ Kavli Institute for Cosmological Physics, University of Chicago, Chicago, IL 60637, USA

${ }^{8}$ Astrophysical Institute, Department of Physics and Astronomy, Ohio University, 251B Clippinger Lab, Athens, OH 45701, USA

${ }^{9}$ Enrico Fermi Institute, Department of Physics, Department of Astronomy \& Astrophysics, and Kavli Institute for Cosmological Physics, University of Chicago, Chicago, IL 60637, USA

${ }^{10}$ Department of Astronomy and Astrophysics, University of California, Santa Cruz, CA 95064, USA

${ }_{11}^{11}$ Astronomy Department, University of Illinois at Urbana-Champaign, 1002 W. Green Street, Urbana, IL 61801, USA

${ }^{12}$ Department of Physics, University of Illinois at Urbana-Champaign, 1110 W. Green Street, Urbana, IL 61801, USA

${ }^{13}$ Steward Observatory, University of Arizona, 933 N. Cherry Avenue, Tucson, AZ 85721, USA

${ }^{14}$ Department of Astronomy \& Astrophysics, Center for Gravitational Wave and Particle Astrophysics, and Center for Theoretical and Observational Cosmology, Pennsylvania State University, 525 Davey Lab, University Park, PA 16802, USA

${ }^{15}$ Instituto de Fisica Teorica UAM/CSIC, Universidad Autonoma de Madrid, E-28049 Madrid, Spain

${ }^{16}$ Kavli Institute for Particle Astrophysics \& Cosmology, P.O. Box 2450, Stanford University, Stanford, CA 94305, USA ${ }^{17}$ SLAC National Accelerator Laboratory, Menlo Park, CA 94025, USA

${ }_{18}^{18}$ National Center for Supercomputing Applications, 1205 West Clark Street, Urbana, IL 61801, USA

${ }^{19}$ Departments of Physics and Astronomy, University of California, Berkeley, CA 94720-3411, USA

${ }^{20}$ Lawrence Berkeley National Laboratory, 1 Cyclotron Road, Berkeley, CA 94720, USA

${ }^{21}$ Center for Cosmology and Particle Physics, New York University, 4 Washington Place, New York, NY 10003, USA

${ }^{22}$ National Optical Astronomy Observatory, 950 North Cherry Avenue, Tucson, AZ 85719, USA

${ }^{23}$ Columbia Astrophysics Laboratory, Columbia University, Pupin Hall, New York, NY 10027, USA

${ }^{24}$ Department of Astronomy \& Theoretical Astrophysics Center, University of California, Berkeley, CA 94720-3411, USA

${ }^{25}$ Space Telescope Science Institute, 3700 San Martin Drive, Baltimore, MD 21218, USA

${ }^{26}$ ICTP South American Institute for Fundamental Research Instituto de Física Teórica, Universidade Estadual Paulista, São Paulo, Brazil

${ }^{27}$ Laboratorio Interinstitucional de e-Astronomia-LIneA, Rua Gal. Jose Cristino 77, Rio de Janeiro, RJ_20921-400, Brazil

${ }^{28}$ Cerro Tololo Inter-American Observatory, National Optical Astronomy Observatory, Casilla 603, La Serena, Chile

${ }^{29}$ Department of Physics \& Astronomy, University College London, Gower Street, London WC1E 6BT, UK

${ }^{30}$ Department of Physics and Electronics, Rhodes University, P.O. Box 94, Grahamstown, 6140, South Africa

${ }^{31}$ Department of Astrophysical Sciences, Princeton University, Peyton Hall, Princeton, NJ 08544, USA

32 Department of Physics and Wisconsin IceCube Particle Astrophysics Center, University of Wisconsin, Madison-Madison, WI 53706, USA ${ }^{33}$ CNRS, UMR 7095, Institut d'Astrophysique de Paris, F-75014 Paris, France

${ }^{34}$ Sorbonne Universités, UPMC Univ Paris 06, UMR 7095, Institut d'Astrophysique de Paris, F-75014 Paris, France

${ }^{35}$ Observatório Nacional, Rua Gal. José Cristino 77, Rio de Janeiro, RJ-20921-400, Brazil

${ }^{36}$ Institut de Ciències de l'Espai, IEEC-CSIC, Campus UAB, Carrer de Can Magrans, s/n, E-08193 Bellaterra, Barcelona, Spain

${ }^{37}$ Institut de Física d'Altes Energies (IFAE), The Barcelona Institute of Science and Technology, Campus UAB, E-08193 Bellaterra (Barcelona) Spain

38 Institute of Cosmology \& Gravitation, University of Portsmouth, Portsmouth PO1 3FX, UK

${ }^{39}$ School of Physics and Astronomy, University of Southampton, Southampton SO17 1BJ, UK

${ }^{40}$ Faculty of Physics, Ludwig-Maximilians-Universität, Scheinerstr. 1, D-81679 Munich, Germany

${ }^{41}$ Excellence Cluster Universe, Boltzmannstr. 2, D-85748 Garching, Germany

${ }^{42}$ Department of Astronomy, University of Michigan, Ann Arbor, MI 48109, USA

${ }^{43}$ Department of Physics, University of Michigan, Ann Arbor, MI 48109, USA

${ }^{44}$ Institute of Astronomy, University of Cambridge, Madingley Road, Cambridge CB3 OHA, UK

${ }^{45}$ Kavli Institute for Cosmology, University of Cambridge, Madingley Road, Cambridge CB3 OHA, UK

${ }_{47}^{46}$ Department of Astronomy, University of California, Berkeley, 501 Campbell Hall, Berkeley, CA 94720, USA

${ }^{47}$ Center for Cosmology and Astro-Particle Physics, The Ohio State University, Columbus, OH 43210, USA 


$$
\begin{gathered}
{ }^{48} \text { Department of Physics, The Ohio State University, Columbus, OH 43210, USA } \\
49 \text { Australian Astronomical Observatory, North Ryde, NSW 2113, Australia } \\
{ }^{50} \text { Departamento de Física Matemática, Instituto de Física, Universidade de São Paulo, CP 66318, CEP 05314-970, São Paulo, SP, Brazil } \\
{ }^{51} \text { George P. and Cynthia Woods Mitchell Institute for Fundamental Physics and Astronomy, and Department of Physics and Astronomy, Texas A\&M University, } \\
\text { College Station, TX 77843, USA } \\
52 \text { Institució Catalana de Recerca i Estudis Avançats, E-08010 Barcelona, Spain } \\
{ }^{53} \text { Max Planck Institute for Extraterrestrial Physics, Giessenbachstrasse, D-85748 Garching, Germany } \\
{ }^{54} \text { Jet Propulsion Laboratory, California Institute of Technology, 4800 Oak Grove Drive, Pasadena, CA 91109, USA } \\
55 \text { Department of Physics and Astronomy, Pevensey Building, University of Sussex, Brighton BN1 9QH, UK } \\
56 \text { Centro de Investigaciones Energéticas, Medioambientales y Tecnológicas (CIEMAT), Madrid, Spain } \\
{ }^{57} \text { Universitäts-Sternwarte, Fakultät für Physik, Ludwig-Maximilians Universität München, Scheinerstr. 1, D-81679 München, Germany } \\
\text { Received 2016 June 10; revised 2016 July 8; accepted 2016 July 9; published 2016 July 29 }
\end{gathered}
$$

\begin{abstract}
We report the results of a Dark Energy Camera optical follow-up of the gravitational-wave (GW) event GW151226, discovered by the Advanced Laser Interferometer Gravitational-wave Observatory detectors. Our observations cover $28.8 \mathrm{deg}^{2}$ of the localization region in the $i$ and $z$ bands (containing 3\% of the BAYESTAR localization probability), starting $10 \mathrm{hr}$ after the event was announced and spanning four epochs at 2-24 days after the GW detection. We achieve $5 \sigma$ point-source limiting magnitudes of $i \approx 21.7$ and $z \approx 21.5$, with a scatter of $0.4 \mathrm{mag}$, in our difference images. Given the two-day delay, we search this area for a rapidly declining optical counterpart with $\gtrsim 3 \sigma$ significance steady decline between the first and final observations. We recover four sources that pass our selection criteria, of which three are cataloged active galactic nuclei. The fourth source is offset by 5.8 arcsec from the center of a galaxy at a distance of $187 \mathrm{Mpc}$, exhibits a rapid decline by 0.5 mag over 4 days, and has a red color of $i-z \approx 0.3 \mathrm{mag}$. These properties could satisfy a set of cuts designed to identify kilonovae. However, this source was detected several times, starting 94 days prior to GW151226, in the Pan-STARRS Survey for Transients (dubbed as PS15cdi) and is therefore unrelated to the GW event. Given its long-term behavior, PS15cdi is likely a Type IIP supernova that transitioned out of its plateau phase during our observations, mimicking a kilonova-like behavior. We comment on the implications of this detection for contamination in future optical follow-up observations.
\end{abstract}

Key words: binaries: close - catalogs - gravitational waves - stars: neutron - surveys

\section{INTRODUCTION}

The Advanced Laser Interferometer Gravitational-wave Observatory (LIGO) is designed to detect the final inspiral and merger of compact object binaries comprised of neutron stars (NSs) and/or stellar-mass black holes (BHs; Abbott et al. 2009). The first LIGO observing run (designated O1) commenced on 2015 September 18 with the ability to detect binary neutron star (BNS) mergers to an average distance of $\approx 75 \mathrm{Mpc}$, a 40-fold increase in volume relative to the previous generation of ground-based gravitational-wave (GW) detectors (The LIGO Scientific Collaboration et al. 2016). On 2015 September 14, LIGO detected the first GW event ever observed, GW150914 (Abbott et al. 2016a).

The waveform of GW150914 was consistent with the inspiral, merger, and ring-down of a binary black hole (BBH) system $\left(36+29 M_{\odot}\right.$; Abbott et al. 2016a) providing the first observational evidence that such systems exist and merge. While there are no robust theoretical predictions for the expected electromagnetic (EM) counterparts of such a merger, more than 20 teams conducted a wide range of follow-up observations spanning from radio to $\gamma$-rays, along with neutrino follow-up (Abbott et al. 2016b; Adrián-Martínez et al. 2016; Annis et al. 2016; Connaughton et al. 2016; Evans et al. 2016; Kasliwal et al. 2016; Savchenko et al. 2016; Smartt et al. 2016; Soares-Santos et al. 2016; Tavani et al. 2016). This effort included deep optical follow-up observations by our group using Dark Energy Camera (DECam) covering $100 \mathrm{deg}^{2}$ (corresponding to a contained probability of $38 \%(11 \%)$ of the initial (final) sky maps) - making this one of the most

\footnotetext{
${ }^{58}$ NSF GRFP Fellow.
}

comprehensive optical follow-up campaigns for GW150914 (Annis et al. 2016; Soares-Santos et al. 2016). Our search for rapidly declining transients to limiting magnitudes of $i \approx 21.5 \mathrm{mag}$ for red $(i-z=1)$ and $i \approx 20.1 \mathrm{mag}$ for blue $(i-z=-1)$ events yielded no counterpart to GW150914 (Soares-Santos et al. 2016). One result of the broader multiwavelength follow-up campaign is a claimed coincident detection of a weak short gamma-ray burst (SGRB) from the Fermi-GBM detector $0.4 \mathrm{~s}$ after the $\mathrm{GW}$ event (Connaughton et al. 2016). However, this event was not detected in INTEGRAL $\gamma$-ray data (Savchenko et al. 2016) and was also disputed in a re-analysis of the GBM data (Greiner et al. 2016).

A second high-significance GW event, designated GW151226, was discovered by LIGO on 2015 December 26 at 03:38:53 UT (Abbott et al. 2016c). This event was also due to the inspiral and merger of a BBH system, consisting of 14.2 $2_{-3.7}^{+8.3} M_{\odot}$ and $7.5_{-2.3}^{+2.3} M_{\odot}$ BHs at a luminosity distance of $d_{L}=440_{-190}^{+180} \mathrm{Mpc}$ (Abbott et al. 2016c). The initial localization was provided as a probability sky map via a private GCN circular $38 \mathrm{hr}$ after the GW detection (LIGO Scientific Collaboration \& Virgo 2015). We initiated optical follow-up observations with DECam $10 \mathrm{hr}$ later on 2015 December 28 and imaged a $28.8 \mathrm{deg}^{2}$ region in the $i$ and $z$ bands during several epochs. Here, we report the results of this search. In Section 2, we discuss the observations and data analysis procedures. In Section 3, we present our search methodology for potential counterparts to GW151226 and the results of this search. We summarize our conclusions in Section 4. We perform cosmological calculations assuming $H_{0}=67.8 \mathrm{~km} \mathrm{~s}^{-1}$ $\mathrm{Mpc}^{-1}, \Omega_{\lambda}=0.69$, and $\Omega_{m}=0.31$ (Planck Collaboration et al. 2015). Magnitudes are reported in the AB system. 
Table 1

Summary of DECam Observations of GW151226

\begin{tabular}{|c|c|c|c|c|c|c|c|c|}
\hline Visit & UT & $\begin{array}{c}\Delta t^{\mathrm{a}} \\
\text { (days) }\end{array}$ & $\begin{array}{c}\left\langle\mathrm{PSF}_{i}\right\rangle \\
(\operatorname{arcsec})\end{array}$ & $\begin{array}{c}\left\langle\mathrm{PSF}_{z}\right\rangle \\
(\operatorname{arcsec})\end{array}$ & $\langle$ airmass $\rangle$ & $\begin{array}{c}\left\langle\operatorname{depth}_{i}\right\rangle \\
(\mathrm{mag})\end{array}$ & $\begin{array}{c}\left.\operatorname{depth}_{z}\right\rangle \\
(\mathrm{mag})\end{array}$ & $\begin{array}{c}A_{\mathrm{eff}^{\mathrm{b}}} \\
\left(\mathrm{deg}^{2}\right)\end{array}$ \\
\hline \multirow[t]{2}{*}{ Epoch 1} & $2015-12-28.11$ & 1.96 & 0.97 & 0.99 & 1.95 & 22.39 & 22.23 & 14.4 \\
\hline & $2015-12-29.11$ & 2.96 & 1.00 & 0.97 & 1.78 & 22.57 & 22.46 & 14.4 \\
\hline Epoch 2 & 2016-01-01.06 & 5.91 & 0.95 & 0.90 & 1.57 & 21.37 & 21.06 & 28.8 \\
\hline \multirow[t]{2}{*}{ Epoch 3} & 2016-01-08.11 & 12.96 & 1.68 & 1.62 & 2.15 & 22.09 & 21.70 & 24.0 \\
\hline & 2016-01-09.11 & 13.96 & 1.17 & 1.12 & 1.80 & 22.44 & 22.17 & 4.8 \\
\hline \multirow[t]{2}{*}{ Epoch 4} & 2016-01-18.03 & 22.88 & 1.21 & 1.20 & 1.48 & 22.00 & 22.01 & 12.0 \\
\hline & 2016-01-19.01 & 23.86 & 1.29 & 1.25 & 1.71 & 21.86 & 21.90 & 16.8 \\
\hline
\end{tabular}

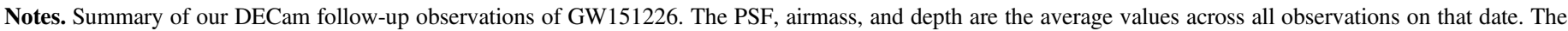
reported depth corresponds to the mean $5 \sigma$ point-source detection in the coadded search images.

a Time elapsed between the GW trigger time and the time of the first image.

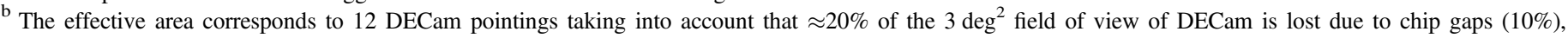
three dead CCDs (5\%; Diehl et al. 2014), and masked edge pixels (5\%).

\section{OBSERVATIONS AND DATA REDUCTION}

GW151226 was detected on 2015 December 26 at 03:38:53 UT by a Compact Binary Coalescence (CBC) search pipeline Abbott et al. (2016c). The CBC pipeline operates by matching the strain data against waveform templates and is sensitive to mergers containing NSs and/or BHs. The initial sky map was generated by the BAYESTAR algorithm and released $38 \mathrm{hr}$ after the GW detection. BAYESTAR is a Bayesian algorithm that generates a localization sky map based on the parameter estimation from the CBC pipeline (Singer et al. 2014; Singer \& Price 2016). The sky area contained within the initial 50\% and $90 \%$ contours was $430 \mathrm{deg}^{2}$ and $1340 \mathrm{deg}^{2}$, respectively. A sky map generated by the LALInference algorithm, which computes the localization using Bayesian forward-modeling of the signal morphology (Veitch et al. 2015), was released on 2016 January 15 UT, after our DECam observations had been concluded. The LALInference sky map is slightly narrower than the sky map from BAYESTAR with 50\% and $90 \%$ contours of $362 \mathrm{deg}^{2}$ and $1238 \mathrm{deg}^{2}$, respectively.

We initiated follow-up observations with DECam on 2015 December 28 UT, two days after the GW detection and 10 hours after distribution of the BAYESTAR sky map. DECam is a wide-field optical imager with a $3 \mathrm{deg}^{2}$ field of view (Flaugher et al. 2015). We imaged a $28.8 \mathrm{deg}^{2}$ region corresponding to $3 \%$ of the sky localization probability when convolved with the initial BAYESTAR map and $2 \%$ of the localization probability in the final LALInference sky map. The pointings and ordering of the DECam observations were determined using the automated algorithm described in SoaresSantos et al. (2016). The choice of observing fields was constrained by weather, instrument availability, and the available time to observe this sky region given its high airmass. We obtained four epochs of data with each epoch consisting of one $90 \mathrm{~s}$ exposure in the $i$-band and two $90 \mathrm{~s}$ exposures in the $z$-band for each of the 12 pointings. The first epoch was obtained 2-3 days after the GW event time (2015 December 28-29 UT), the second epoch was at 6 days (2016 January 1 UT), the third epoch was at 13-14 days (2016 January 8-9), and the fourth epoch was at 23-24 days (2016 January 18-19). A summary of the observations is provided in Table 1, and a visual representation of the sky region is shown in Figure 1.

We processed the data using an implementation of the photpipe pipeline modified for DECam images. Photpipe

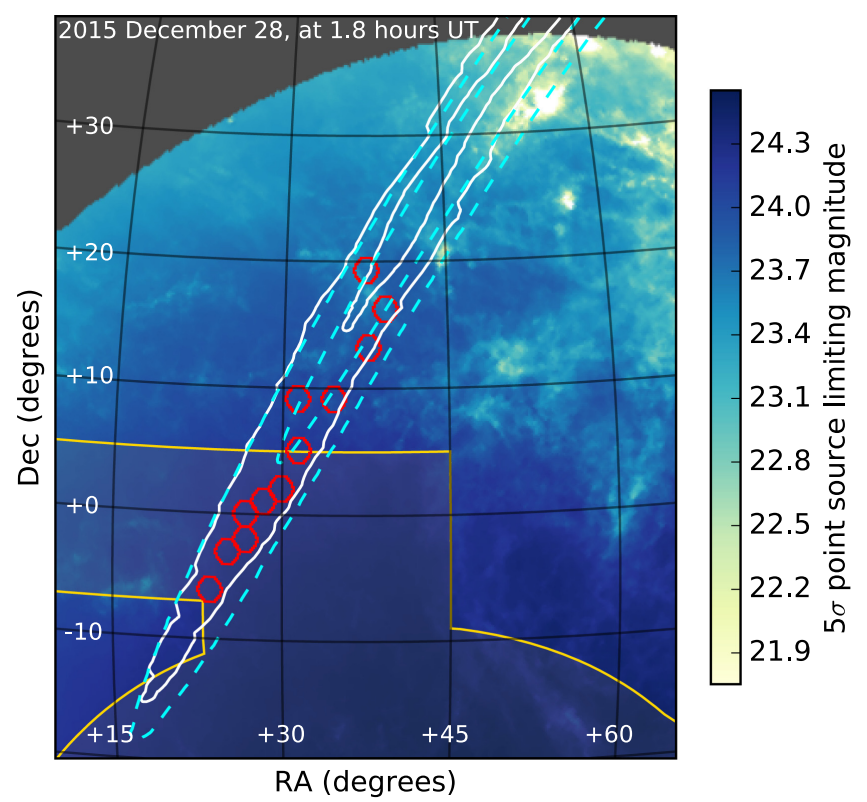

Figure 1. Sky region covered by our DECam observations (red hexagons) relative to the $50 \%$ and $90 \%$ probability regions from the BAYESTAR (cyan contours) and LALInference (white contours) localization of GW151226. The background color indicates the estimated $5 \sigma$ point-source limiting magnitude for a $90 \mathrm{~s} i$-band exposure as a function of sky position for the first night of our DECam observations. The variation in the limiting magnitude is largely driven by the dust extinction and airmass at that position. The dark gray regions indicate sky positions that were unobservable due to the telescope pointing limits. The yellow contour indicates the region of sky covered by the Dark Energy Survey (DES). The total effective area for the 12 DECam pointings is $28.8 \mathrm{deg}^{2}$, corresponding to $3 \%(2 \%)$ of the probability in the BAYESTAR (LALInference) sky map.

is a pipeline used in several time-domain surveys (e.g., SuperMACHO, ESSENCE, Pan-STARRS1; see Rest et al. 2005; Garg et al. 2007; Miknaitis et al. 2007; Rest et al. 2014), designed to perform single-epoch image processing including image calibration (e.g., bias subtraction, cross-talk corrections, flat fielding), astrometric calibration, image coaddition, and photometric calibration. Additionally, photpipe performs difference imaging using hotpants (Alard 2000; Becker 2015) to compute a spatially varying convolution kernel, followed by photometry on the difference images using an implementation of DoPhot optimized for point-spread function (PSF) photometry on difference images (Schechter et al. 
1993). Last, we use photpipe to perform initial candidate searches by specifying a required number of spatially coincident detections over a range of time. Once candidates are identified, photpipe performs "forced" PSF photometry on the subtracted images at the fixed coordinates of an identified candidate in each available epoch.

In the case of the GW151226 observations, we began with raw images acquired from the NOAO archive ${ }^{59}$ and the most recent calibration files. ${ }^{60}$ Astrometric calibration was performed relative to the Pan-STARRS1 (PS1) $3 \pi$ survey and 2MASS $J$-band catalogs. The two $z$-band exposures were then coadded. Photometric calibration was performed using the PS1 $3 \pi$ survey with appropriate calibrations between PS1 and DECam magnitudes (Scolnic et al. 2015). Image subtraction was performed using observations from the final epoch as templates. The approach to candidate selection is described in Section 3.

Our observations achieved average $5 \sigma$ point-source limiting magnitudes of $i \approx 22.2$ and $z \approx 21.9$ in the coadded singleepoch search images, and $i \approx 21.7$ and $z \approx 21.5$ in the difference images, with an epoch-to-epoch scatter of 0.4 mag. The variability in depth is driven by the high airmass and changes in observing conditions, particularly during the second epoch.

\section{SEARCH FOR AN OPTICAL COUNTERPART}

The primary focus of our search is a fast-fading transient. While the merger of a BBH system is not expected to produce an EM counterpart, it is informative to consider the possibility of optical emission due to the presence of some matter in the system. As a generic example, we consider the behavior of a transient such as an SGRB with a typical beaming-corrected energy of $E_{j} \approx 10^{49} \mathrm{erg}$ and an opening angle of $\theta_{j} \approx 10^{\circ}$ (Berger 2014; Fong et al. 2015). If viewed far off-axis $\left(\theta_{\text {obs }} \gtrsim 4 \theta_{j}\right)$, the optical emission will reach peak brightness after several days, but at the distance of GW151226 $(\approx 440 \mathrm{Mpc}$; Abbott et al. 2016c), the peak brightness will be $i \approx 26$ mag (see Figure 5 of Metzger \& Berger 2012), well beyond our detection limit. If the source is observed moderately off-axis or on-axis $\left(\theta_{\text {obs }} \lesssim 2 \theta_{j}\right)$, then the light curve will decline throughout our observations, roughly as $F_{\nu} \propto t^{-1}$, and will be detectable at $i \approx 21-22$ mag in our first observation (see Figures 3 and 4 of Metzger \& Berger 2012). We can apply a similar argument to the behavior of a more isotropic (and non-relativistic) outflow given that any material ejected in a $\mathrm{BBH}$ merger is likely to have a low mass and the outflow will thus become optically thin early, leading to fading optical emission. Based on this line of reasoning, we search our data for steadily declining transients.

We identify relevant candidates in the data using the following selection criteria with the forced photometry from photpipe. Unless otherwise noted, these criteria are applied to the $i$-band data due to the greater depth in those observations.

1. We require non-negative or consistent with zero (i.e., within $2 \sigma$ of zero) $i$ - and $z$-band fluxes in the difference photometry across all epochs to eliminate any sources

\footnotetext{
59 http://archive.noao.edu/

${ }^{60}$ http://www.ctio.noao.edu/noao/content/decam-calibration-files
}

that re-brighten in the fourth (template) epoch. This provides an initial sample of 602 candidates.

2. We require $\geqslant 5 \sigma i$ - and $z$-band detections in the first epoch and at least one additional $\geqslant 5 \sigma i$-band detection in either of the two remaining epochs (to eliminate contamination from asteroids). This criterion leaves a sample of 98 objects.

3. We require $a \geqslant 3 \sigma$ decline in flux between the first and third epochs to search for significant fading. ${ }^{61} \mathrm{We}$ calculate $\sigma$ as the quadrature sum of the flux errors from the first and third epochs $\left(\sigma=\sqrt{\sigma_{1}^{2}+\sigma_{3}^{2}}\right.$, where $\sigma_{1}$ and $\sigma_{3}$ are the flux errors from the first and third epochs, respectively). This criterion leaves a sample of 48 objects.

4. We reject sources that exhibit a significant $(\geqslant 3 \sigma)$ rise in flux between the first and second epochs or the second and third epochs to eliminates variable sources that do not decline steadily. This criterion leaves a sample of 32 objects.

5. The remaining 32 candidates from step 4 undergo visual inspection. We reject sources that are present as a point source in the fourth (template) epoch that do not have a galaxy within $20^{\prime \prime}$. Sources are cross-checked against NED $^{62}$ and SIMBAD. ${ }^{63}$ This criterion is designed to remove variable stars and long-timescale transients.

Only four events passed our final criterion. We find that two of those events are coincident with the nuclei of known active galactic nuclei (AGNs; PKS 0129-066 and Mrk 584), indicating that they represent AGN variability. A third candidate is coincident with the nucleus of the bright radio source PMN J0203+0956 $\left(F_{\nu}(365 \mathrm{MHz}) \approx 0.4 \mathrm{Jy}\right.$; Douglas et al. 1996), also suggesting AGN variability.

The final candidate in our search is located at R.A. $=01^{\mathrm{h}} 42^{\mathrm{m}} 16^{\mathrm{s}} .17$ and decl. $=-02^{\circ} 13^{\prime} 426^{\prime \prime}(\mathrm{J} 2000)$, with an offset of 5.8 arcsec from the galaxy CGCG 386-030 (R.A. $=01^{\mathrm{h}} 42^{\mathrm{m}} 15^{\mathrm{s}} .6, \quad$ decl. $=-02^{\circ} 13^{\prime} 385^{\prime \prime} ; \quad$ J2000), at $z=0.041$ or $d_{L} \approx 187 \mathrm{Mpc}$ (6dFGS; Jones et al. 2004, 2009; see Figure 2). We note that this distance is inconsistent with the $90 \%$ confidence interval for the distance to GW151226 based on the GW data (Abbott et al. 2016c). We observe this source in a state of rapid decline with an absolute magnitude of $M_{i} \approx-15 \mathrm{mag}$ on 2015 December 28 and $M_{i} \approx-14.5 \mathrm{mag}$ on 2016 January 1 , indicating a decline rate of $\approx 0.12 \mathrm{mag} \mathrm{d}^{-1}$; the decline rate in the $z$-band is $\approx 0.10 \mathrm{mag}$ $\mathrm{d}^{-1}$. Additionally, the source exhibits a red $i-z$ color of $0.3 \mathrm{mag}$. We fit these data to a power-law model typical for GRB afterglows $\left(F_{\nu} \propto \nu^{\beta} t^{\alpha}\right)$ and find a temporal index of $\alpha=-0.43 \pm 0.12$ and a spectral index of $\beta=-1.8 \pm 0.8$, both of which differ from the expected values for GRB afterglows ( $\alpha \approx-1, \beta \approx-0.75$; Sari et al. 1998). Additionally, we compare our observations to a kilonova model with ejecta parameters of $v_{\mathrm{ej}}=0.2 \mathrm{c}$ and $M_{\mathrm{ej}}=0.1 M_{\odot}$ (Barnes \& Kasen 2013). We find that the timescale of the transient agrees with those expected for kilonovae, but the color is bluer than the expected value of $i-z \approx 1$ mag (Barnes \& Kasen 2013).

\footnotetext{
61 We note that this criterion effectively requires the detection in the first epoch to be $\geq 5 \sigma$, producing an effectively shallower transient search. Soares-Santos et al. (2016) quantified this effect by injecting fake sources into their observations to determine the recovery efficiency and loss of detection depth from analysis cuts. Here, we forego such analysis to focus the discussion on the effects of contamination in optical follow-up of GW events.

62 https://ned.ipac.caltech.edu/

63 http://simbad.u-strasbg.fr/simbad/
} 


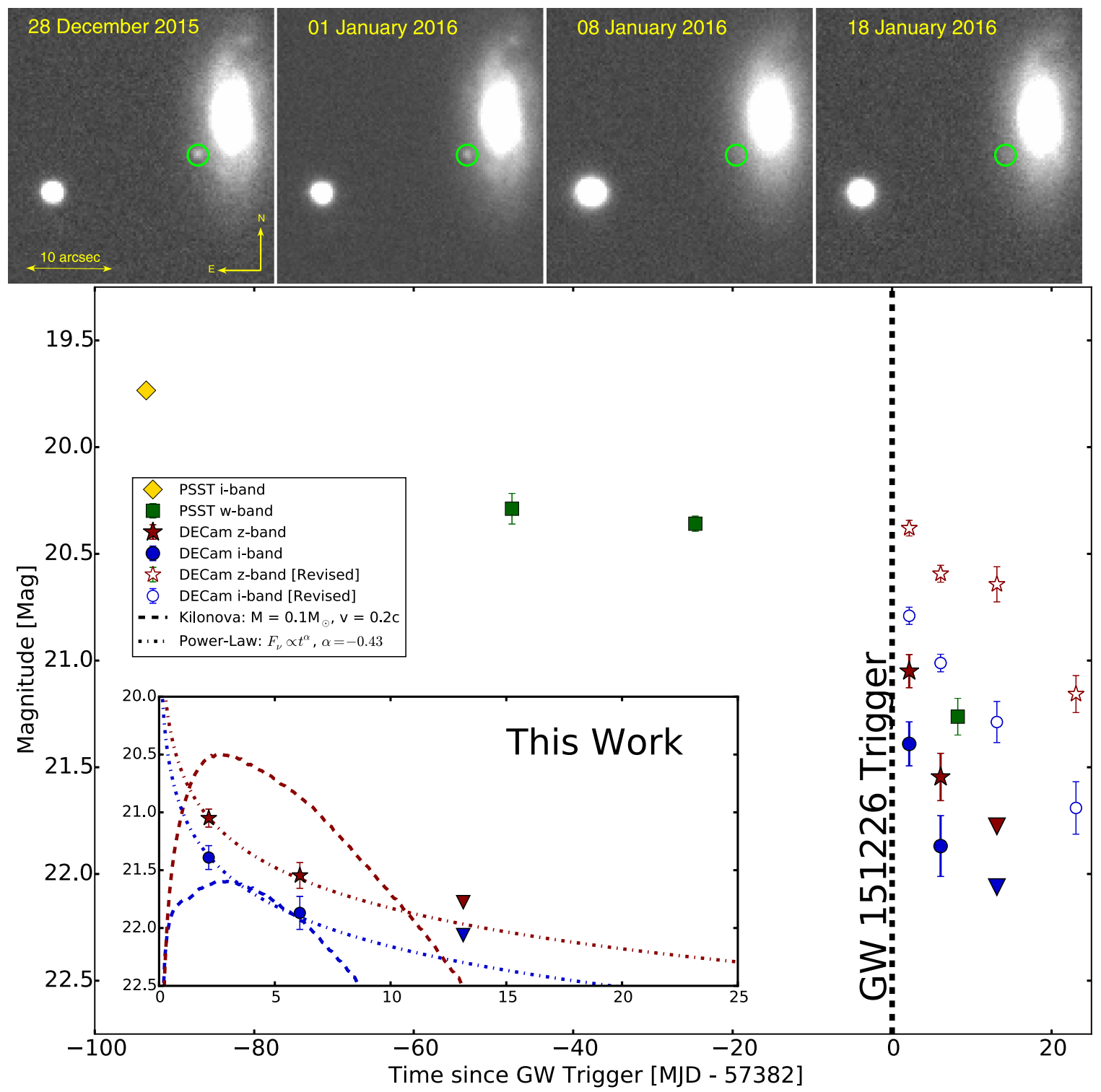

Figure 2. Top: single-epoch images of our main candidate from all four epochs (green circle). This is the event discovered as PS15cdi in the PSST about 94 days prior to GW151226. Bottom: light curve data for PS15cdi from PSST $w$ - and $i$-band observations (green squares and yellow diamonds, respectively). Our DECam $i$ - and $z$-band data are shown as blue circles and red stars, respectively. The revised DECam analysis using pre-existing templates is shown as open symbols. Upper limits are indicated by triangles. The inset focuses on our DECam data, indicating a rapid decline in both the $i$ and $z$ bands. We fit a power-law model to the data finding a temporal index of $\alpha=-0.43$ (dashed-dotted line). Kilonova models from Barnes \& Kasen (2013) with $v_{\mathrm{ej}}=0.2 c$ and $M_{\mathrm{ej}}=0.1 M_{\odot}$ at a distance of $187 \mathrm{Mpc}$ are also shown (dashed line).

Thus, the properties of this transient differ from those of GRB afterglows or kilonovae. The observations and models are shown in Figure 2.

This source was previously detected as PS15cdi on 2015 September 23 by the Pan-STARRS Survey for Transients (PSST $^{64}$; Huber et al. 2015; see Figure 2). The absolute $i$-band magnitude in the first PSST epoch, $M_{i} \approx-16.6 \mathrm{mag}$, and the shallow decline of $\approx 0.6$ mag over $\approx 70 \mathrm{~d}$ are consistent with a Type IIP core-collapse supernova (SN). A likely interpretation of the rapid decline in our observations is that PS15cdi is a Type IIP SN undergoing the rapid transition from the hydrogen recombination driven plateau to the radioactive ${ }^{56} \mathrm{Co}$-dominated phase (Kasen \& Woosley 2009; Sanders et al. 2015;

\footnotetext{
$\overline{64}$ http://psweb.mp.qub.ac.uk/ps1threepi/psdb/candidate/ 1014216170021342600/
}

Dhungana et al. 2016). The red $i-z$ color in our data is consistent with observations of other IIP SN during this phase of evolution (e.g., SN2013ej; Dhungana et al. 2016). This transition typically occurs about 100 days post-explosion (Kasen \& Woosley 2009; Sanders et al. 2015; Dhungana et al. 2016), consistent with the timing of our observations relative to the first detection in PSST.

To mitigate the effect of excess flux from PS15cdi still present in our template observations, we repeat the analysis using as templates archival DES $i$ - and $z$-band images from 2013 December 19. These data were processed and image subtraction was performed as described in Section 2. We find that flux from PS15cdi is indeed still present in our original template image, leading to revised first epoch absolute magnitudes of $M_{i} \approx-15.6$ and $M_{z} \approx-16 \mathrm{mag}$, and a decline rate between the first and fourth epochs of $0.04 \mathrm{mag} \mathrm{d}^{-1}$, in 
both the $i$ and $z$ bands. The transient still exhibits a red $i-z$ colors of $\approx 0.4$ mag across all four epochs.

Clearly, we can rule out this candidate based on the PSST detections prior to GW151226, but without this crucial information this candidate would have been a credible optical counterpart based on its light curve behavior and distance. It is therefore useful to develop an understanding of the expected rates for such contaminants to inform expectations in future searches. We adopt a local core-collapse $\mathrm{SN}$ rate of $7 \times 10^{-5} \mathrm{yr}^{-1} \mathrm{Mpc}^{-3}$ (Li et al. 2011; Cappellaro et al. 2015) and a Type IIP SN fraction of $48 \%$ of this rate (Smith et al. 2011). The rapid decline phase typically lasts about 20 days (Kasen \& Woosley 2009; Sanders et al. 2015; Dhungana et al. 2016), so we consider events that occur within that time frame. Last, given its apparent brightness, we assume that PS15cdi represents the approximate maximum distance to which we can observe these events in our data. We thus find an expected occurrence rate of $\sim 0.04$ events in our search area making our detection of PS15cdi somewhat unlikely and indicating that $\lesssim 1$ such events are expected in a typical GW localization region.

Our detection of PS15cdi clearly demonstrates the presence and impact of contaminants when conducting optical follow-up of GW events. Core-collapse SNe are generally not considered to be a significant contaminant due to their much longer timescales compared to kilonovae (e.g., Cowperthwaite \& Berger 2015). However, a source like PS15cdi, caught in a rapid phase of its evolution despite its overall long timescale and exhibiting a relatively red color, could satisfy a set of criteria designed for finding kilonovae $\left(\Delta m \gtrsim 0.1 \mathrm{mag} \mathrm{d}^{-1}\right.$ and $i-z \gtrsim 0.3 \mathrm{mag}$; Cowperthwaite \& Berger 2015).

The most effective approach to dealing with contaminants like PS15cdi is rapid, real-time identification. Once a candidate is deemed interesting, optical spectroscopy and NIR photometry can quickly distinguish between an SN or a kilonova/ afterglow origin. Specifically, the kilonova spectrum will be redder, with clear suppression below $\sim 6000 \AA$ due to the opacities of $r$-process elements (Kasen et al. 2013). By comparison, the SN spectrum will appear bluer and dominated by iron group opacities (Kasen et al. 2013), while the afterglow spectrum will exhibit a featureless power-law spectrum (Berger 2014). If pre-existing templates are not available, then the significant aspect is rapid initiation of follow-up observations at $\lesssim 1$ day that can distinguish the rising phase of a kilonova or off-axis GRB from a declining SN.

\section{CONCLUSIONS}

We presented the results of our deep optical follow-up of GW151226 using the DECam wide-field imager. Our observations cover a sky area of $28.8 \mathrm{deg}^{2}$, corresponding to $3 \%$ of the initial BAYESTAR probability map and $2 \%$ of the final LALInference map. We obtained four epochs of observations starting $10 \mathrm{hr}$ after the event was announced and spanning 2-24 days post-trigger, with an average $5 \sigma$ point-source sensitivity of $i \approx 21.7$ and $z \approx 21.5$, with an epoch-to-epoch scatter of $0.4 \mathrm{mag}$, in our difference images.

Using the final epoch as a template image, we searched for sources that display a significant and steady decline in brightness throughout our observations and that are not present in the template epoch. This search yielded four transients, of which three result from AGN variability. The final event is located at a distance of about $187 \mathrm{Mpc}$ offset by 5". 8 from its host galaxy. It also broadly possesses the observational features of a kilonova in terms of its rapid decline and red $i-z$ color. However, this source corresponds to the transient PS15cdi, which was discovered in PSST about 94 days prior to the GW trigger. It is a likely Type IIP SN, which our observations caught in the steep transition at the end of the plateau phase. The detection of this event indicates that careful rejection of contaminants, preferably in real time, is essential in order to avoid misidentifications of optical counterparts to GW sources.

P.S.C. is grateful for support provided by the NSF through the Graduate Research Fellowship Program, grant DGE1144152. R. J.F. gratefully acknowledges support from NSF grant AST1518052 and the Alfred P. Sloan Foundation. D.E.H. was supported by NSF CAREER grant PHY-1151836. He also acknowledges support from the Kavli Institute for Cosmological Physics at the University of Chicago through NSF grant PHY1125897 as well as an endowment from the Kavli Foundation.

This research uses services or data provided by the NOAO Science Archive. NOAO is operated by the Association of Universities for Research in Astronomy (AURA), Inc. under a cooperative agreement with the National Science Foundation. The computations in this Letter were run on the Odyssey cluster supported by the FAS Division of Science, Research Computing Group at Harvard University. This research has made use of the NASA/IPAC Extragalactic Database (NED), which is operated by the Jet Propulsion Laboratory, California Institute of Technology, under contract with the National Aeronautics and Space Administration. Light curve data for PS15cdi were obtained from The Open Supernova Catalog (Guillochon et al. 2016). Some of the results in this Letter have been derived using the HEALPix package (Górski et al. 2005).

Funding for the DES Projects has been provided by the DOE and NSF (USA), MEC/MICINN/MINECO (Spain), STFC (UK), HEFCE (UK). NCSA (UIUC), KICP (U. Chicago), CCAPP (Ohio State), MIFPA (Texas A\&M), CNPQ, FAPERJ, FINEP (Brazil), DFG (Germany) and the Collaborating Institutions in the Dark Energy Survey.

The Collaborating Institutions are Argonne Lab, UC Santa Cruz, University of Cambridge, CIEMAT-Madrid, University of Chicago, University College London, DES-Brazil Consortium, University of Edinburgh, ETH Zürich, Fermilab, University of Illinois, ICE (IEEC-CSIC), IFAE Barcelona, Lawrence Berkeley Lab, LMU München and the associated Excellence Cluster universe, University of Michigan, NOAO, University of Nottingham, Ohio State University, University of Pennsylvania, University of Portsmouth, SLAC National Lab, Stanford University, University of Sussex, Texas A\&M University, and the OzDES Membership Consortium.

The DES Data Management System is supported by the NSF under grant number AST-1138766. The DES participants from Spanish institutions are partially supported by MINECO under grants AYA2012-39559, ESP2013-48274, FPA2013-47986, and Centro de Excelencia Severo Ochoa SEV-2012-0234. Research leading to these results has received funding from the ERC under the EU's 7th Framework Programme including grants ERC 240672, 291329, and 306478.

\section{REFERENCES}

Abbott, B. P., Abbott, R., Abbott, T. D., et al. 2016a, PhRvL, 116, 061102 Abbott, B. P., Abbott, R., Abbott, T. D., et al. 2016b, ApJL, 826, L13 Abbott, B. P., Abbott, R., Abbott, T. D., et al. 2016c, PhRvL, 116, 241103 
Abbott, B. P., Abbott, R., Adhikari, R., et al. 2009, RPPh, 72, 076901 Adrián-Martínez, S., Albert, A., André, M., et al. 2016, PhRvD, 93, 122010 Alard, C. 2000, A\&AS, 144, 363

Annis, J., Soares-Santos, M., Berger, E., et al. 2016, ApJL, 823, L34

Barnes, J., \& Kasen, D. 2013, ApJ, 775, 18

Becker, A. 2015, HOTPANTS: High Order Transform of PSF And Template Subtraction, Astrophysics Source Code Library, ascl:1504.004

Berger, E. 2014, ARA\&A, 52, 43

Cappellaro, E., Botticella, M. T., Pignata, G., et al. 2015, A\&A, 584, A62

Connaughton, V., Burns, E., Goldstein, A., et al. 2016, ApJL, 826, L6

Cowperthwaite, P. S., \& Berger, E. 2015, ApJ, 814, 25

Dhungana, G., Kehoe, R., Vinko, J., et al. 2016, ApJ, 822, 6

Diehl, H. T., Abbott, T. M. C., Annis, J., et al. 2014, Proc. SPIE, 9149, 91490V

Douglas, J. N., Bash, F. N., Bozyan, F. A., Torrence, G. W., \& Wolfe, C. 1996, AJ, 111,1945

Evans, P. A., Kennea, J. A., Barthelmy, S. D., et al. 2016, MNRAS, 460, L40

Flaugher, B., Diehl, H. T., Honscheid, K., et al. 2015, AJ, 150, 150

Fong, W., Berger, E., Margutti, R., \& Zauderer, B. A. 2015, ApJ, 815, 102

Garg, A., Stubbs, C. W., Challis, P., et al. 2007, AJ, 133, 403

Górski, K. M., Hivon, E., Banday, A. J., et al. 2005, ApJ, 622, 759

Greiner, J., Burgess, J. M., Savchenko, V., \& Yu, H.-F. 2016, ApJL, in press (arXiv:1606.00314)

Guillochon, J., Parrent, J., \& Margutti, R. 2016, ApJ, submitted (arXiv:1605. 01054)

Huber, M., Carter Chambers, K., Flewelling, H., et al. 2015, IAUGA, 22, 2258303

Jones, D. H., Saunders, W., Colless, M., et al. 2004, MNRAS, 355, 747
Jones, D. H., Read, M. A., Saunders, W., et al. 2009, MNRAS, 399, 683

Kasen, D., Badnell, N. R., \& Barnes, J. 2013, ApJ, 774, 25

Kasen, D., \& Woosley, S. E. 2009, ApJ, 703, 2205

Kasliwal, M. M., Cenko, S. B., Singer, L. P., et al. 2016, ApJL, 824, L24

Li, W., Chornock, R., Leaman, J., et al. 2011, MNRAS, 412, 1473

LIGO Scientific Collaboration \& Virgo 2015, GCN Circ., 18728

Metzger, B. D., \& Berger, E. 2012, ApJ, 746, 48

Miknaitis, G., Pignata, G., Rest, A., et al. 2007, ApJ, 666, 674

Planck Collaboration, Ade, P. A. R., Aghanim, N., et al. 2015, arXiv:1502. 01589

Rest, A., Scolnic, D., Foley, R. J., et al. 2014, ApJ, 795, 44

Rest, A., Stubbs, C., Becker, A. C., et al. 2005, ApJ, 634, 1103

Sanders, N. E., Soderberg, A. M., Gezari, S., et al. 2015, ApJ, 799, 208

Sari, R., Piran, T., \& Narayan, R. 1998, ApJL, 497, L17

Savchenko, V., Ferrigno, C., Mereghetti, S., et al. 2016, ApJL, 820, L36

Schechter, P. L., Mateo, M., \& Saha, A. 1993, PASP, 105, 1342

Scolnic, D., Casertano, S., Riess, A., et al. 2015, ApJ, 815, 117

Singer, L. P., \& Price, L. R. 2016, PhRvD, 93, 024013

Singer, L. P., Price, L. R., Farr, B., et al. 2014, ApJ, 795, 105

Smartt, S. J., Chambers, K. C., Smith, K. W., et al. 2016, arXiv:1602.04156

Smith, N., Li, W., Filippenko, A. V., \& Chornock, R. 2011, MNRAS, 412, 1522

Soares-Santos, M., Kessler, R., Berger, E., et al. 2016, ApJL, 823, L33

Tavani, M., Pittori, C., Verrecchia, F., et al. 2016, ApJL, 825, L4

The LIGO Scientific Collaboration, Martynov, D. V., Hall, E. D., et al. 2016, PhRvD, 93, 112004

Veitch, J., Raymond, V., Farr, B., et al. 2015, PhRvD, 91, 042003 